
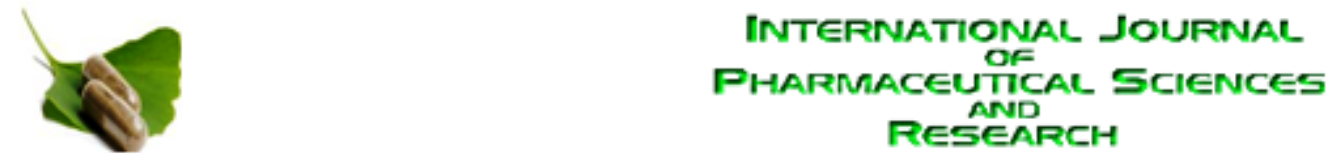

Received on 22 September, 2012; received in revised form, 17 October, 2012; accepted, 17 December, 2012

\title{
CONFORMATIONAL ANALYSIS: A REVIEW
}

Jasmine Uthuppan ${ }^{1}$ and Kriti Soni ${ }^{2}$

Department of Pharmaceutical Chemistry ${ }^{1}$, Department of Pharmaceutics ${ }^{2}$, Faculty of Pharmacy, Jamia Hamdard, Hamdard Nagar, New Delhi-110062, India

Keywords:

Algorithm, Cartesian Coordinates,

Conformations, Cluster Analysis,

Optimization, Root-Mean-Square Distance, Torsional Angle

Correspondence to Author:

Jasmine Uthuppan

Department of Pharmaceutical Chemistry, Faculty of Pharmacy, Jamia Hamdard, Hamdard Nagar, New Delhi-110062, India

E-mail: jasmine239d@yahoo.com

\section{ABSTRACT}

Conformational analysis is an important step in molecular modeling as it is necessary to reduce time spent in screening of compounds for activity. Most drugs are flexible molecules with the ability to adopt different conformations by means of rotation about single bonds. Conformations play an important role in prediction of not just physico-chemical properties but also the biological activity of the drug. This review details the various methods involved in conformational analysis. The major objective of conformational analysis is to gain insight on conformational characteristic of drugs and also to identify the relation between the role of conformational flexibility and their activity.
INTRODUCTION: The most important concerns in Medicinal chemistry and pharmaceutical research are structure elucidation, conformational analysis, physicochemical characterization and biological activity determination. The determination of molecular structure is essential as the structure of the molecule predicts the physical, chemical, and biological properties of the molecule ${ }^{1}$.

Conformational search methods find applications in the design of targeted chemical hosts and drug discovery ${ }^{2}$. Conformations are different 3D spatial arrangements of the atoms in a molecule are interconvertible by free rotation of single bonds ${ }^{3}$.

The major objective of conformational analysis is to gain insight on conformational characteristic of flexible biomolecules and drugs but to also identify the relation between the role of conformational flexibility and their activity. Therefore, it plays a significant role in computer aided design as well. The significance of conformational analysis not just extends to computational docking and screening but also for lead optimization ${ }^{4}$.
Conformational Analysis: DHR Barton is considered the most important contributor to modern conformational analysis. In 1950, he showed how various substituents at the equatorial and axial positions affect the rate of reactivity of substituted cyclohexanes. Identification of all possible minimumenergy structures (conformations) of a molecule is the goal of conformational analysis ${ }^{5}$.

Conformational analysis is a computational method in which restraints are used such that the molecule presumes a conformation similar to the rigid template molecule. Conformational analysis is a difficult problem because even simple molecules may have a large number of conformational isomers ${ }^{5}$. The usual strategy in conformational analysis is to use a search algorithm to generate a series of initial conformations.

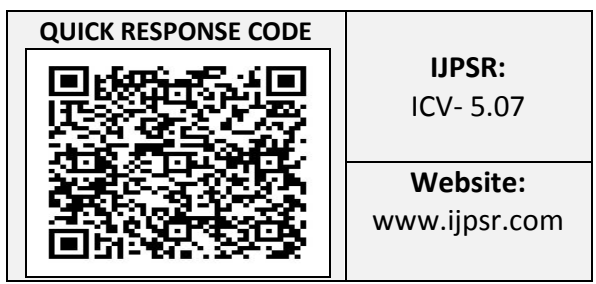


Each of these in turn are then subjected to energy minimization in order to derive the associated minimum energy structure [Leach 1991, 2001; Scheraga 1993] ${ }^{6}$. Global minimum-energy conformation is the conformation with the lowest energy. It is not imperative that the global energy minimum conformation is the bioactive conformation of the drug
${ }^{5}$. Most drugs being flexible molecules can by means of distortions and rotations about rotable bonds adopt large number of conformations. Pharmacophore is a collection of steric and electronic features that are essential to ensure optimal communication between the specific biological targets (Receptor/Enzyme) so as to illict a biological response.

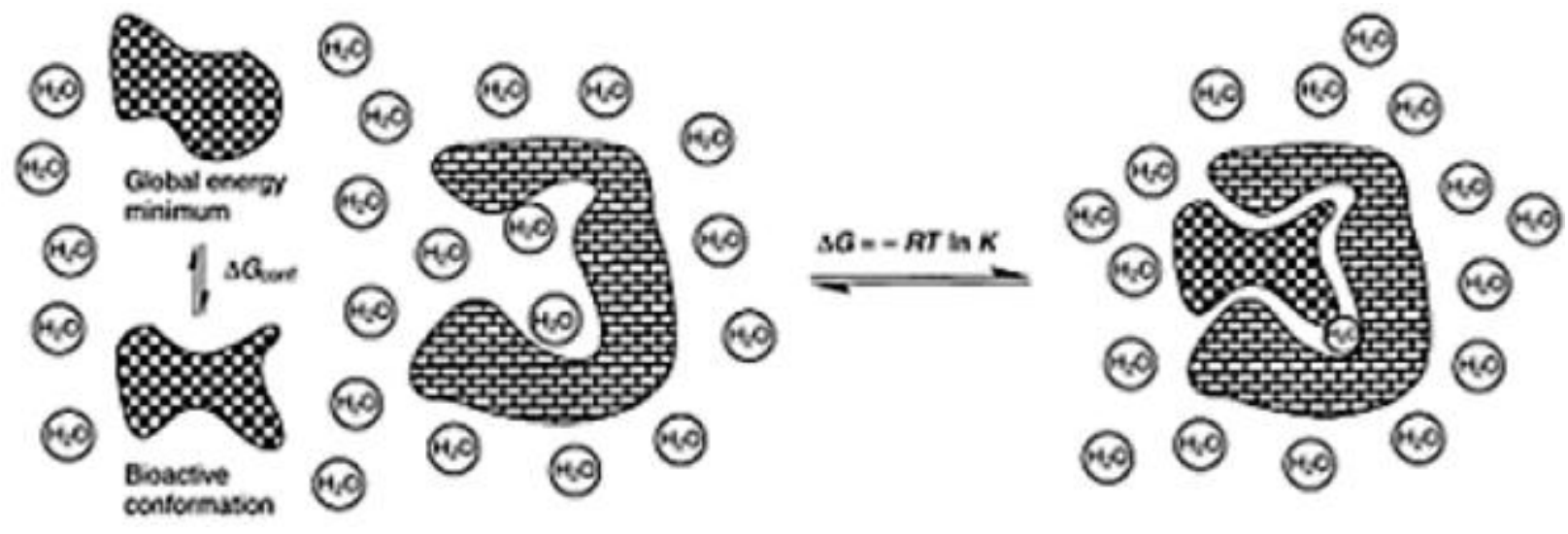

FIG 1: THE EQUILIBRIUM CHARACTERIZING THE RECEPTOR LIGAND COMPLEX INTERACTION

[Larsen P.K., Liljefors T \& Madsen U. in "Textbook of Drug Design \& Drug Discovery",..3 ${ }^{\text {rd }}$ ed. New York: Taylor \& Francis, 2005, 103]

The most difficult and main problem in 3D pharmacophore recognition is the identification of the receptor bound/bioactive conformation. This can be achieved by studies of the spatial arrangement of the bioactive conformer so as to define the 3D pharmacophore ${ }^{7}$. This conformation could be the local minimum, global minimum or any transition state between the local minima. Although it is common practice to assume that global minimum is the bioactive conformation.

Different methods used to determine information regarding conformations is ${ }^{3}$.

- X-ray Diffraction

- Electron Diffraction

- Raman Spectroscopy,

- UltraViolet Spectroscopy

- InfraRed Spectroscopy

- NMR Spectroscopy

- Microwave Spectroscopy

- Photoelectron Spectroscopy

- Supersonic Molecular Jet Spectroscopy

- Optical Rotatory Dispersion and CD measurements.

- Molecular Mechanics

\section{Overview:}

1. Conformational Sampling

A. Systematic Search Method

B. Random Searches

C. Metropolis Monte Carlo Methods

D. Genetic Algorithm and Evolutionary Algorithm

E. Model Building Methods

F. Molecular Dynamics

G. Distance geometry

H. Neural Networks

2. Conformation Optimization

3. Conformational analysis

1. Conformational Sampling: Conformation sampling is a procedure used to create a collection of molecular conformations that will later be analyzed. Ideally, all locally stable conformations of the molecule should be reported for in order for 
the conformational analysis to be complete. As the size of the molecule increases, the number of locally stable conformations increases so fast that the task of full enumeration becomes formidable. The basic prerequisite is that the resulting conformational sample "ensemble" will be representative of the system as a whole ${ }^{4}$.

A. Systematic Search method: In this method, there is systemic generation of conformations and thus exploration of the conformational space through assigning of discrete values to torsion angles of rotatable bonds of the drug which results in regular and predictable alterations of the structure. The values are limited to a set of predetermined values. In systematic search, exploration of the energy surface of the molecule is carried out in a predictable pattern. It is used where it's not possible to predict the order in which conformations will be generated by random methods ${ }^{5}$.

Grid search: Is the simplest type of systematic search algorithm in which conformations corresponding to all possible combinations of torsion angle values are generated. Series of conformations are generated by systematic rotation of torsion angles around the rotatable single bonds between angles of $0^{\circ}$ and $360^{\circ}$.

For example, If a molecule has two variable torsion angles such that the first torsion angle can adopt the values $60^{\circ}, 180^{\circ}$ and $-60^{\circ}$ and the second torsion angle may adopt values $0^{\circ}$ and $180^{\circ}$. Six conformations will be generated by the grid search (i.e. $60^{\circ}, 0^{\circ} ; 60^{\circ}, 180^{\circ} ; 180^{\circ}, 0^{\circ} ; 180^{\circ}, 180^{\circ} ;-60^{\circ}, 0^{\circ}$; $\left.-60^{\circ} ; 180^{\circ}\right)^{6}$. The number of conformations is given by;

$$
\text { Number of conformations }=\mathrm{S}^{N}
$$

Where $N$ is the number of free rotation angles, $S$ is the number of discrete values for each rotation angle.

$$
S=360 / \vartheta i
$$

$\vartheta i$ being the dihedral increment of angle $i$.
Due to the exponential increase in number of conformations increases due to the number of bonds that have free rotation lead to combinatorial complexity, which is most crucial drawback involved in a systematic search. Building molecules from aggregates or by the use of distance constraint equations are some methods that help in resolving the combinatorial explosion ${ }^{1}$.

B. Random Searches: Random search is the exact opposite of systematic search. It can move from one region of the conformational space to a completely unconnected new region in a single step. Random search methods can study conformational spaces either changing the atomic cartesian coordinates or torsion angles of rotable bonds ${ }^{5}$.

A number of random conformational search methods have been devised [Saunders 1987; Li and Scheraga 1987; Ferguson and Raber 1989; Chang et al., 1989]. They all generally involve a repetitive procedure in which an initial structure is selected, randomly modified and minimized ${ }^{6}$.

At each step random change is made to the current conformer found and structure is refined using energy minimization. The conformation generated is added to the list of structures found. This conformation is starting point for next step and the sequence is repeated ${ }^{5}$.

Since there is no natural end point; the process continues until either a predefined number of iterations have been attempted and/or until no new conformations are generated ${ }^{6}$.

\section{RANDOM SEARCH}

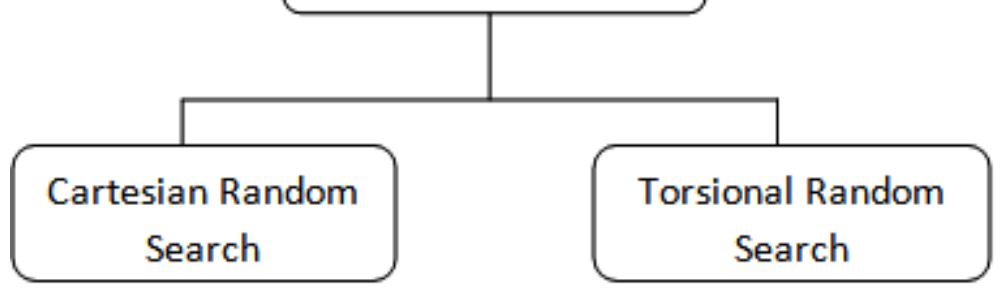


DIFFERENCES BETWEEN CARTESIAN RANDOM SEARCH AND TORSIONAL RANDOM SEARCH ${ }^{5}$

$\begin{array}{ccc}\text { S. No. } & \text { Cartesian Random Search } & \text { Torsional Random Search } \\ \text { 1. } & \begin{array}{c}\text { Addition of random amount to x, y and z coordinates } \\ \text { of all atoms in a molecule occurs. [Saunders 1987; } \\ \text { Ferguson \& Raber 1989] }\end{array} & \begin{array}{c}\text { Random change to torsion angles of rotable bonds are made such that } \\ \text { bond lengths and bond angles are kept fixed. } \\ \text { [Li \& Scheraga 1987; Chang et al 1987] }\end{array} \\ \text { 2. } & \begin{array}{c}\text { Implementation is simple but relies deeply on energy } \\ \text { minimization as structures generated can be } \\ \text { disoriented or have extremely high energy. }\end{array} & \begin{array}{c}\text { Special procedures are needed to change torsional angles in molecules } \\ \text { containing rings as ring constraints must be fulfilled in each ring. }\end{array}\end{array}$

C. Monte Carlo Method: Monte Carlo Method is one of the important methods as it was the technique used to execute the first computer stimulation of a molecular system ${ }^{5}$. The method is based on statistical mechanics. Monte Carlo Minimum method (MCMM) of Chang et al is where "A single starting geometry is used repeatedly to accumulate its progeny" 8 . An important feature of this method is that is that the Monte Carlo scheme can study, sample and calculate energy of all the states. The Boltzmann weight contribution to the average can also be calculated. On the other hand, one can ignore states that have high energy and which contribute little weight, if any, weight to average and concentrate on low energy structures ${ }^{8}$.

The method introduces a prejudice towards stable conformations. This is termed as Importance Sampling. The parameter coordinates are changed at each step so as to achieve maximum effectiveness.

In Simple Stochastic search, from a given starting structure random alterations are made and internal energy of resulting structure is calculated and energy minimization is undertaken. The new conformer produces is saved if the energy or the difference in energies between the new and the best conformer is less than the threshold optimum value.

$$
\begin{gathered}
\Delta \mathrm{E}<\Delta \mathrm{E}_{\mathrm{MIN}} \\
\Delta \mathrm{E}-\mathrm{E}_{\mathrm{MIN}}<\Delta \mathrm{E}
\end{gathered}
$$

The search is automatically concluded after a user defined number of unproductive attempts. The termination number is set at about a few hundred $^{8}$.
The MC method has been successfully used to find minimum energy conformations for cycloalkanes using specific constraint conditions, but cannot generally be extended to unrestricted systems ${ }^{1}$. Monte Carlo method is appropriate for large molecules with complex interconversion pathways ${ }^{8}$.

\section{Systematic Unbounded Minimum Method} (SUMM): It is a variant of the MC method. The SUMM approach of Goodman and Still suggests a new internal coordinate transversal of conformational space. Low resolution (120 ${ }^{0}$ Torsion angle space) generates torsionally remote conformers early on in the search method. It appears to be more efficient at finding low energy conformations for medium and large ring molecules ${ }^{8}$.

Random Incremental Pulse Search (RIPS): In this method starting with input geometry, random changes $\left( \pm 1 A^{0}\right)$ is preformed on the coordinates of each atom or a subset of atoms and the perturbed geometry is then subjected to minimization to find new conformers ${ }^{8}$.

D. Metropolis Monte Carlo Method: The approach amplifies the chances of finding the global minimum. It involves number of sequences where the Monte Carlo Algorithm is run at different temperatures. The first phase runs at temperature $T_{1}$ and an assortment of conformations are generated. The most stable conformation is used as starting origin for next phase where temperature is set at lower temperature. The process is repeated until the probability equation becomes selective towards which structure is accepted. Thus a small part of the conformation space is meticulously investigated. 
Similar to stochastic method it introduces a probabilistic criteria to accept new conformers. The Boltzmann factor is calculated using the formula

$$
P=\exp ^{-\Delta E / K b T}
$$

Where $\mathrm{P}$ - Probability Criteria, $\Delta \mathrm{E}$ Energy factor, $K$ is the Boltzmann constant, $T$ is similar to temperaturein Boltzmann Law and is termed temperature by analogy.

The acceptance test is performed by choosing a random number (between 0 and 1 ) and evaluating it against the probability factor ( $\exp ^{-\Delta E /}$ $\mathrm{Kb}^{\mathrm{T}}$ ). If the random number is lower, the change is accepted and new conformation is yielded which can be used as the new starting point.

Also If the Boltzmann factor is larger than a random number between zero and one then the new structure is selected. If not then the previous structure is retained ${ }^{9}$.

Genetic Algorithm: Genetic algorithms (GAs) are a class of optimization methods that are based on various computational models of Darwinian evolution [Goldberg 1989] ${ }^{6}$.A Genetic Algorithm is a large-scale optimization algorithm mimicking a biological evolution in a randomly generated population. A number of conformations form this population. The adaptation is calculated, and a new population is created in accordance to operators (crossover, and mutation). The process is repeated until it converges to a minimum energy structure ${ }^{1}$.

Selection of the initial population of conformers analogous to parent is carried out with a statistical bias such that only stable conformations are selected. The chromosome represented by torsional angles or any other parameter may alter due to crossover or mutation resulting in new and diversified conformers. The process may be repeated for as long as it is practically possible.

Stable configurations may be formed early and can be lost due to crossover or mutation. To prevent this most programs have an elitist strategy to carry forward the most stable conformations ${ }^{9}$.
Evolutionary Algorithm: It is slightly different from genetic algorithm as no crossover takes place in this process. Only Mutations are used to generate new conformations. The first and second generations undergo a competition to see which represents the stable conformations. It is analyzed by comparison of each conformer with another randomly chosen one. The stable conformations that score more are preserved and low score conformations are rejected 9 .

E. Model Building Approaches: The assumptions of the Model Building Approach are:-

- Each of the fragments must be conformationally independent of the other fragments of the molecule.

- Each fragment conformations stored must cover range of structures that are observed in the completely built molecule.

In this model construction of conformation of the molecule is done by fusion of three dimensional structures of molecular fragments. It is anticipated to be more effective than normal systematic search as the number of combinations of fragment conformations is less than that of the combination of torsional angles or Cartesian coordinates. The program must decide which fragments are required to construct the molecule [Leach et al., 1990]. This is accomplished by utilizing the sub culture search algorithm, which determines whether each of fragments that program knows about is present and how they match onto the atoms in the molecule ${ }^{5}$.

F. Molecular dynamics: Molecular dynamics (MD) is a conformational space search procedure in which the atoms of molecule are given an initial velocity and are then allowed to evolve in time according to the laws of Newtonian mechanics ${ }^{10}$. In this method, generation of successive configurations is carried out by incorporation of Newton's law of motion for theatoms in the system, to provide a trajectory that defines how the positions and velocities of theparticles of the system vary with time. 
Molecular dynamics is a computer program that treats the atoms within the molecules as moving spheres. After $10^{-15} \mathrm{sec}$ of movement, determination of the position and velocity of each atom in structure is used for the estimation of the forces by utilizing the values of bond lengths, bond angles, torsional terms and nonbonded interactions. The calculation of potential energy of each atom and Newton's Law of Motion helps in the computation of acceleration and direction of each atom.

Generation of different conformations is carried out by program by "Heating" of molecule which implies that the molecule undergoes bond stretching and bond rotation as if it was being heated. The process can be repeated automatically to give any number of practical structures ${ }^{9}$. Molecular Dynamics thus provides not only information about the conformation system but also the way in which the conformation changes with time ${ }^{5}$. Twist boat conformation of cyclohexane is not the most stable form but molecular dynamic program predicts a more stable chair form ${ }^{9}$.

G. Distance geometry: Distance geometry uses the interatomic distances and various mathematical procedures to generate structures of conformations for energy minimization ${ }^{6}$. Aszodi et al. explored the use of distance geometry as the metric for comparative modeling of structures ${ }^{12}$. In this method, a set of distance constraints are transformed into a set of Cartesian coordinates ${ }^{1}$.

The crucial feature is that it is not possible to randomly assign interatomic distance values in a molecule and low energy conformations are formed as a result of this feature ${ }^{5}$. The distances are calculated according to bond angles, and torsion angles for free and rigid angles, and any other known constraints on the system. These distances are taken into a matrix and lower and upper bounds are represented ${ }^{1}$. Derivation of a conformations by Distance geometry occurs in four stages. [Crippen 1981; Crippen and Havel 1988]
1. Matrix containing the maximum and minimum values permitted to each interatomic distance in the molecule is calculated.

2. Each interatomic distance is arbitrarily assigned values between the upper and lower bounds.

3. Distance Matrix is transformed into trial set of cartesian coordinates.

4. Refinement of structure and generation of conformation is the last stage.

Refinement of structure is carried out in accordance to simple trigonometric restrictions ${ }^{5}$. The distance between $A$ and $C$ can be no greater than the sum of maximum values of distances between $A B$ \& $B C$.

$$
\mathrm{U}_{\mathrm{AC}} \leq \mathrm{U}_{\mathrm{AB}}+\mathrm{U}_{\mathrm{BC}}
$$

Minimum value of $A C$ distance can be no less than the difference between the lower bound on $A B$ and the upper bound on $B C$ :

$$
L_{A C} \geq L_{A B}-U_{B C}
$$

The distance matrix is subjected to a process called embedding, In which the distance space is representation of conformation is converted to a set of atomic Cartesian coordinates. This generates the conformation ${ }^{5}$. The method is applicable to conformational search on small or medium size molecules.

H. Neural Networks: Artificial neural networks are based on the concepts that were motivated by the theories of the cellnetwork of the human brain. Neural networks are designed tomimic the information processing and knowledge acquisition of the human brain. They have basic segments called artificial neurons that perform identical tasks ${ }^{11}$. They are generally applied to nonlinear problems and pattern recognition studies. In conformational analysis, neural networks have been used to predict the maximumand minimum distances between pairs of heteroatoms ${ }^{1}$. 
It is used in studies related to protein folding, secondary structure, location of disulfide bridges and surface accessible of each residue.

2. Conformational Optimization: The various sampling procedures result in a number of transient conformations. During energy minimization of these structures is to bring these conformations to the minima before analysis is performed. Minimization methods are used as a tool in model building, preparation of the initial structure for molecular dynamics and groundwork of structures for normal mode analysis ${ }^{4}$.

Stimulated annealing [Kirkpatrick et al., 1983] ${ }^{6}$ is used in optimization problems where minimization of objective function can be carried out without being caught in local minima. It is designed for finding the global minimum and samples low energy conformations more sharply. It is derived by analogy with the process of crystallization; slow cooling produces stable crystalline forms corresponding to global minimum, whereas rapid freezing may lead to the formation of metastable form corresponding to local minimum.

Stimulated annealing is monitored by a cooling schedule and the process is broken up into a number of cycles, each at constant temperature and composed of large number of individual levels corresponding to stochastic search with Metropolis criterion. Each level is believed to be producing Boltzmann distribution.

The temperature is lowered and process is repeated. Alternatively it can be said that heating up the system provides the molecule the energy facilitating it to leap out of its initial local minimum. The gradual cooling that is consequently applied decreases the amplitude of these vibrations and conveys the particulars of the energy surface back into focus and causes the system to gradually settle down to a lower energy minimum ${ }^{4}$. Due to progressive decrease in temperature explorations of conformations of decreasing cost can analyze smaller and smaller regions of conformational space such that system falls into global minimum ${ }^{8}$.
3. Conformational Analysis: The analysis of the conformational collection that was sampled and optimized is essential so as to ascertain the conformational properties of the molecule that is being studied. This helps to underline the global properties and to exemplify features of overall flexibility and to recognize common inclination in the conformation set. Alternatively, it may be used to identify a smaller subset of characteristic low energy conformations, which may be used to direct future drug development efforts ${ }^{4}$.

A. Similarity Measures: A similarity measure is essential for quantitative comparison of one structure with another and it must be defined before the analysis. Structural similarity is calculated by a root-mean-square distance (RMSD) between two conformations. In Cartesian coordinates the RMS distance dij between conformation $i$ and conformation $j$ of a given molecule is defined as the minimum of the functional

$$
d_{i j}=\left[\frac{1}{N} \sum_{k=1}^{N}\left|\mathbf{r}_{k}^{(i)}-\mathbf{r}_{k}^{(j)}\right|^{2}\right]^{1 / 2}
$$

Where, $N$ is the number of atoms in the summation, $k$ is an index over these atoms, $r_{k}{ }^{(i)}, r_{k}{ }^{(i j)}$ are the Cartesian coordinates of atom $k$ in conformations $i$ and j.

B. Cluster Analysis: Cluster analysis is a regular investigative technique used to group conformations. In this method structural similarity are highlighted and are defined by the distance measure being used within a conformational sample. The initial conformation selected is generally of low energy and all conformations that are contained by a given cutoff distance from this structure are grouped together to form first cluster. The ungrouped conformations are selected and new cluster is formed. The procedure is repeated until all conformations are assigned into clusters. In many conformational studies, cluster analysis is used as a way to focus futureeffort on a small set of characteristic conformations. 
One conformation, typically the lowestenergy one, is picked from each of the highly populated conformational clusters. Theresulting small number of distinctly different conformations are then used as starting pointsfor further computational analysis (such as free energy simulations) or as a basis for generatinga pharmacological hypothesis used for directing future drug development ${ }^{4}$.

\section{Applications:}

- Used in the refinement of X-ray and NMR data to determine the three dimensional structures of large biological molecules such as protein, automated docking of substrates to proteins and thus in protein engineering.

- The generation of Quantitative Structure-Activity Relationships (QSAR) models.

- Receptor modeling, particularly regarding the binding of small ligand molecules to sites of proteins or macromolecules. .

- Use of Conformational Restriction in drug designThe constrained systematic search procedure was used in the discovery of 3D pharmacophores for the inhibition of Angiotensin Converting Enzyme (ACE), an enzyme involved in the regulation of blood pressure [Dammkoehler et al., 1989].

- Design and analysis of compound library.

CONCLUSION: This review is a description of the various computational procedures that are involved in conformational analysis. The methods for sampling, optimization and analysis of conformations have been discussed. In practice, many variations of the basic methodologies are used by research scientists so as to increase the efficiency of processes and reduce development time. Different combinations of these methods can also be used in combination to perform complete conformational analysis of any molecule ${ }^{4}$.
With enormous generation of data and increased synthesis and isolation of new compounds, conformational analysis is an essential part so that systemic interpretation and representation of data is made possible.

Thus this review gives the reader a better insight into the strategies used in conformational analysis. Conformational analysis, due to its wide range of applications forms a pivotal part in modern drug design.

\section{REFERENCES}

1. Bruni A.T, Leite B. P Vitor, Ferreira M. C. Marcia: Conformational Analysis: A New Approach by Means of Chemometrics, John Wiley \& Sons, Inc. 2002, DOI 10.1002/jcc.1004.

2. Cheng L, Cai W and Shao X : A Conformational Analysis Method for Understanding the Energy Landscapes of Clusters , ChemPhysChem 2007, 8, 569 - 577 @ 2007 Wiley-VCH Verlag GmbH \& Co. KGaA, Weinheim, DOI: 10.1002/cphc.200600604.

3. Smith.B.M \& March.J, March's Advanced Organic Chemistry: Reactions, Mechanisms, and Structure, sixth edition 2007, John Wiley \& Sons Inc, 195-196.

4. Becker. $\mathrm{O}$,MacKerellJr.A , Roux.B and Watanabe.M Marcel Dekker : Computational biochemistry and Biophysics, Inc.270 Madison Avenue, New York, NY 10016, 2001, 69-85

5. Leach A. R: Molecular Modelling Principles and Applications, Second Edition 2001, Pearson Education Ltd Essex England, 410, 457-460, 464-469

6. Leach.A.R. \& Gillet.J.V :An Introduction to Chemoinformatics, Revised editon 2007, Springer, AA Dordrecht, The Netherlands; 34-38, 47

7. Larsen P.K, Liljefors T. \& Madsen U : Textbook of Drug Design \& Drug Discovery, $3^{\text {rd }}$ ed. New York: Taylor \& Francis, 2005, 102

8. Doucet Jean-Pierre and Weber.J :Computer-Aided Molecular Design Theory and Applications, 1996, Elsevier Ltd; 206, 209, 212-213

9. Patrick, G.L: An Introduction to Medicinal Chemistry, $4^{\text {th }}$ ed. USA:Oxford University Press, 2009, 342-347.

10. Gupta. R, Tiwari. A and Yadav. M: Molecular dynamics : Basic study, International Journal of Bioinformatics Research, ISSN: 0975-3087 \& E-ISSN: 0975-9115, Volume 4, Issue 1, 2012, 245248.

11. Terfloth.L and Gasteiger.J : Neural networks and genetic algorithms in drug design, 2001 Elsevier Science Ltd. All rights reserved. PII: S1471-1931(01)00173-2, DDT Vol. 6, No. 15 (Suppl.), 2001

12. Abraham J.D: Burger's Medicinal Chemistry, Sixth Edition Volume 1. A John Wiley \&Sons Inc Publications, 96-98,126.

How to cite this article:

Uthuppan J and Soni K: Conformational Analysis: A Review. Int J Pharm Sci Res. 2013; 4(1); 34-41. 